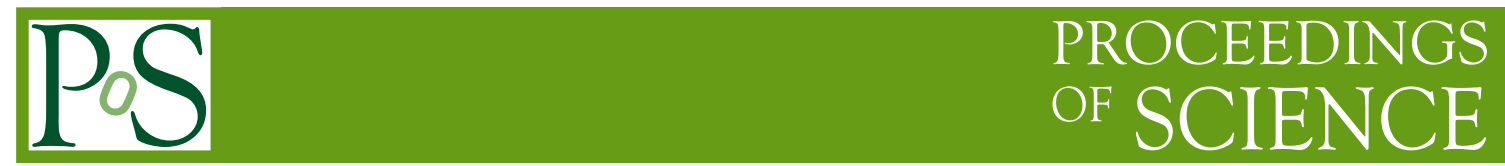

\title{
ATLAS electroweak measurements with early data
}

\author{
Kristin Lohwasser*ifor the ATLAS Collaboration \\ University of Oxford \\ E-mail: kristin.lohwasser@cern.ch
}

\begin{abstract}
The analysis of $W$ and $Z$ production with early data is crucial to the success of the ATLAS experiment. These processes exhibit large cross sections, their precise measurement is needed to maximise the discovery potential of the machine. These proceedings give an overview of early measurements suited to validate and constrain electroweak parameters as well as accompanying studies that will evaluate the performance of the ATLAS detector in-situ.
\end{abstract}

2008 Physics at LHC

September 29 - 4 October 2008

Split, Croatia

*Speaker.

${ }^{\dagger}$ Supported by Deutscher Akademischer Austauschdienst DAAD 


\section{Introduction}

One of the priorities after the start of data taking of the ATLAS detector will be the so-called "re-discovery" of the Standard Model. Measuring theoretically well understood processes such as the production of the electroweak bosons $W$ and $Z$ will enable the validation of the modelling of these processes at the highest centre-of-mass energies. Since $W$ and $Z$ will become backgrounds to many analyses of physics beyond the Standard Model, it is crucial to understand their production early on. The study of $W$ and $Z$ production can also help to understand the detector performance.

These proceedings discuss selected electroweak (EW) measurements, cross section ratios for $W$ and $Z$ production as well as $W$ and $Z$ asymmetries. They also introduce studies on how to determine the most important systematics, including efficiencies as well as missing transverse energy, $\not_{T}$, and lepton energy scales and resolutions, for the presented measurements using early data.

\section{Electroweak Physics Measurements at ATLAS}

The production of the EW bosons $W$ and $Z$ is a clean process with a large cross section: For each lepton flavour, the $Z$ production cross-section times branching ratio, $\sigma(p p \rightarrow Z+X) \times \mathscr{B}(Z \rightarrow$ $\ell \ell$ ), is about $2 \mathrm{nb}$, the one for $\sigma(p p \rightarrow W+X) \times \mathscr{B}(W \rightarrow \ell v)$ with $20 \mathrm{nb}$ a factor of 10 higher. The theoretical predictions are precise: Differential distributions of $W$ and $Z s$ are available in next-tonext-to-leading-order (NNLO) precision with a scale dependence of below 1\% [1]. The analytical QCD calculations yield a precision that requires the inclusion of NLO electroweak corrections which are of the same magnitude. Other theoretical uncertainties arise from the Parton Distribution Functions (PDF), which describe the inital states in hadron collisions. For $W$ production cross section measurements at ATLAS, the error arising from PDFs is 1\% when using CTEQ6.5[2] PDF sets. Cross section measurements can validate these precise predictions, but rely on the determination of the luminosity, which will initially only be known with an uncertainty of 20-30\% [3].

At ATLAS the following robust selection cuts will be used with early data: For the $W$, the lepton and the missing transverse energy are each required to be above $25 \mathrm{GeV}$, the transverse mass of the $W, M_{T}^{W}>40 \mathrm{GeV}$. For $Z$ analyses, the $p_{T}^{\ell}$ cuts are lower, $p_{T}^{\ell}>15 \mathrm{GeV}$. The reconstructed $Z$ mass is constrained to be within a mass window of $20 \mathrm{GeV}$ around the nominal $Z$ mass. Both electrons and muons are generally reconstructed up to pseudorapidities of $|\eta|<2.5$, the maximal coverage of the ATLAS inner tracker, and are required to be isolated.

\section{1 $Z, W$ cross-section ratio}

Measurements of the cross-section ratio of the EW bosons have the advantage that they do not rely on the determination of the luminosity. QCD and EW effects will cancel as will also correlated uncertainties related to efficiencies for one of the leptons in $Z$ and $W$ events, provided they are treated carefully. This will improve the precision that can be accomplished. This makes the measurement of the $W$ and $Z$ cross section ratio attractive for early analysis. It is defined as:

$$
R=\frac{\sigma_{W} \times \mathscr{B}(W \rightarrow \ell v)}{\sigma_{Z} \times \mathscr{B}(Z \rightarrow \ell \ell)}=\frac{\sigma_{W} \Gamma_{Z} \Gamma_{W \rightarrow \ell v}}{\sigma_{Z} \Gamma_{W} \Gamma_{Z \rightarrow \ell \ell}}
$$

Measuring $R$ at higher centre-of-mass energies than at the Tevatron is an interesting analysis as such, but can also provide us with better knowledge of other parameters. Using precise LEP 
results as constraint on $\Gamma_{Z}$ and the EW branching ratios, one can gain access to the width of the $W$, $\Gamma_{W}$. The cross section ratio is very sensitive to differences in efficiencies and acceptances of the two bosons, thus is it essential to understand and model these correctly with early data.

\subsection{Z asymmetry}

The coupling of the $Z$ is a mixture of $\mathrm{V}$ and A couplings, which is controlled by the weak mixing angle $\sin ^{2} \theta_{\mathrm{W}}$. Measuring the amount of parity violation in $Z$ decays at ATLAS gives a handle on the weak mixing angle $\sin ^{2} \theta_{\mathrm{W}}$. This is done by looking at the angular distribution of the decay leptons in $Z \rightarrow e e$ events in the centre-of-mass system of the $e^{+} e^{-}$pair, measured as the scattering angle $\theta^{*}$ between electron and quark. The excess of forward $\left(N_{F}\right.$ with $\left.\cos \theta^{*}>0\right)$ compared to backwards events $\left(N_{B}\right.$ with $\left.\cos \theta^{*}<0\right)$ is an indicator of parity violation. The exact relation of this forward-backward asymmetry $A_{F B}$ to the electroweak mixing angle $\sin ^{2} \theta_{\mathrm{W}}$ is:

$$
A_{F B}=\frac{N_{F}-N_{B}}{N_{F}+N_{B}}=b\left(a-\sin ^{2} \theta_{\mathrm{W}}\right)
$$

Here, $a$ and $b$ are PDF correction parameters. The need for correction arises from the fact that in $p p$ interactions the direction of the incoming quark cannot be directly measured, but is inferred from the $Z$ longitudinal momentum. The boost of the $Z$ along the beam axis is assumed to coincide with the direction of the incoming quark, which causes the longitudinal boost since it generally carries a higher momentum fraction $x$ than the anti-quark with which it annihilates.

With $100 \mathrm{fb}^{-1}$ ATLAS will be able to perform a competitive measurement of $\sin ^{2} \theta_{\mathrm{W}}$ [4]. Analysis with early data allows crucial checks. To enhance the sensitivity, only one of the $Z$ decay leptons is required to fall within the tracker acceptance of $|\eta|<2.5$, while the other is reconstructed using only the calorimeter up to $|\eta|<4.9$. As a consequence, the electron is identified using exclusively the reconstructed charge of the central lepton. This makes it essential to understand the charge reconstruction of the leptons, while uncertainties due to luminosity or acceptance cancel.

\section{$2.3 W$ asymmetry}

One of the first quantities to be measured at ATLAS to improve our knowledge of PDFs is the asymmetry $A$ in the rapidity distributions of $\mathrm{W}^{ \pm}$bosons. It is defined as

$$
A=\frac{d \sigma\left(W^{+}\right) / d \eta-d \sigma\left(W^{-}\right) / d \eta}{d \sigma\left(W^{+}\right) / d \eta+d \sigma\left(W^{-}\right) / d \eta}
$$

$A$ is dependent on the momenta of the partons annihilating and can be inferred from the pseudorapidity $\eta$ distribution of the decay leptons of the $W$. Previous studies using ATLAS simulations have shown, that the $\eta$ distributions and the lepton asymmetry from $W$ s can be used to get improved PDFs compared to PDFs derived from data covering a restricted kinematic range of $x>10^{-3}$ [5], since the $W$ production at high $\eta$ is sensitive to low- $x$ values up to $x \approx 10^{-5}$. Many systematic uncertainties cancel, namely luminosity, QCD and EW corrections as well as efficiencies. An important issue is the background from QCD dijets. 

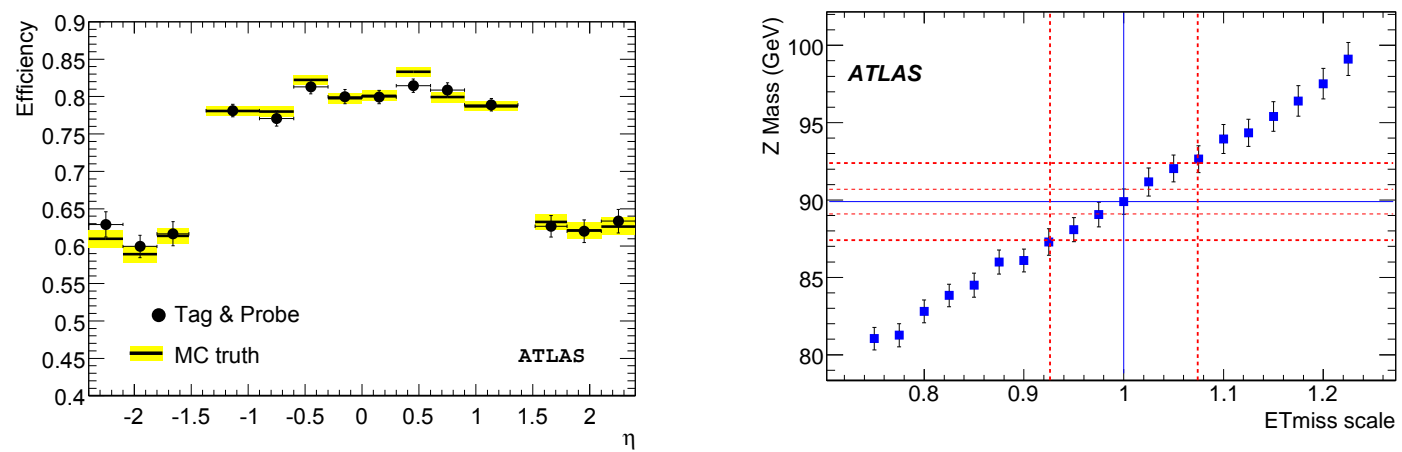

Figure 1: The left plot shows the combined electron efficiency of trigger selection and identification cuts as a function of $\eta$. Compared are tag $\&$ probe as well as MC results for $50 \mathrm{pb}^{-1}$. The right plots shows the dependence of the reconstructed $Z$ mass on the $\not_{T}$ scale for $Z \rightarrow \tau \tau$ events where one of the $\tau$ s decays leptonically.

\section{Understanding the Performance of the ATLAS detector}

Each of the measurements with $W$ and $Z$ bosons presented here, requires a good understanding of the detector with the emphasis on different performance aspects. Fortunately, $W$ and $Z$ bosons can also be used to understand the detector itself using data-driven techniques, reducing reliance on Monte Carlo generators and detector simulation.

\subsection{Lepton efficiencies}

Understanding the efficiencies of lepton triggering and identification is crucial for cross section measurements as well as measurements relying on charge identification. Used in this context is the tag $\&$ probe method. It employs a $Z \rightarrow \ell \ell$ sample, where one of the leptons is used as tag and the other as probe. The tag lepton is required to be triggered and very well identified. The probe lepton is used to test trigger and identification selections. The total efficiency for the early data selection cuts including an electron trigger with a threshold of $20 \mathrm{GeV}$ will be about $80 \%$ for electrons with $|\eta|<1.37$. The systematical and statistical precision of the trigger efficiencies extracted with the tag \& probe method is estimated to be below $2 \%$ with $50 \mathrm{pb}^{-1}$ for muons and electrons [4].

The charge misidentification of leptons due to a wrong track assignment or track misreconstruction can be determined using a similar method, where the number $N$ of well reconstructed $Z \mathrm{~s}$ with like-sign leptons is compared to the number of all reconstructed Zs. Using some refinements, this method can be also applied differentially in $\eta$, where the misidentification is found to be below $0.1 \%$ in the central region and about $1 \%$ for $|\eta|>1.52$ [4].

\section{2 $\not_{T}$ and electron energy scales and resolutions}

The precision of the lepton energy and $Z_{T}$ is a crucial factor in $Z$ and $W$ measurements. It is desirable to determine both, scale and resolution, using data-driven methods in order not to be reliant on detector simulation in early data measurements. Various data-driven methods making use of EW bosons have been tested with simulated ATLAS events. Electron energy scales and resolutions can be determined using $Z \rightarrow e e$ events. The measured $Z$ line shape is compared with the 
expected line shape as measured at LEP. A log-likelihood fit is used to extract scale and resolution from the comparison of the measured line shape and the theoretical expectation. The uncertainty on the results is expected to be better than $0.5 \%$ for scale and resolution for $100-200 \mathrm{pb}^{-1}$ [4].

The extraction of the $Z_{T}$ scale using in-situ measurements will be more challenging. It can be achieved with an scale uncertainty of $8 \%$ using $Z \rightarrow \tau \tau$ sample of $100 \mathrm{pb}^{-1}$, where one of the $\tau$ s decays leptonically. The value of the reconstructed $Z$ mass in these events is very sensitive to the $Z_{T}$ scale, see fig. 1 . The resolution can be extracted using $Z \rightarrow \ell \ell$ events, in which the $Z_{T}$ is projected onto the flight direction of the reconstructed $Z$ [4]. There should be no genuine $Z_{T}-$ the measured $Z_{T}$ depends on the hadronic activity and is caused by mismeasurements and fluctuations.

\subsection{QCD backgrounds from data}

Jets being misidentified as electrons make up a large fraction of the background especially in $W \rightarrow e v$ events. Since there are large uncertainties on the total rate of this background, it is necessary to determine it using data-driven methods. While the background to $Z$ events can be estimated using a fit to the mass peak that convolves a signal and a background function, the datadriven determination of the QCD background for $W \rightarrow e v$ is more complicated. Here, events are selected that reproduce the same $Z_{T}$ shape as the QCD background to the final event selection for a $W$ analysis. For this control sample photons are selected, using identification cuts based on shower shapes in the calorimeter. These cuts are the same as for electrons, however objects identified as photons cannot have an assigned track. There is no source for $\not_{T}$ in prompt photon events, that does not also occur in QCD di-jet events, be it semileptonic heavy flavour decays or genuine fake $Z_{T}$. Hence, the $Z_{T}$ distribution of the selected photon events should reflect the distribution of $Z_{T}$ in fake electron events. The $Z_{T}$ distribution of the photon events can be normalised to the measured $E_{T}$ distribution in a region dominated by background, $10 \mathrm{GeV}<Z_{T}<22.5 \mathrm{GeV}$. After this it is possible to extrapolate the $E_{T}$ distribution and extract the number of expected background events in the signal region. After background subtraction the fraction of remaining QCD background to the $W$ signal was found to be $0 \% \pm 4 \%$ (stat.) [4].

\section{Conclusions}

Various data-driven methods were developed and tested by the ATLAS collaboration in order to be able to access systematic uncertainties for early measurements such as the $W$ and $Z$ cross sections, ratios and asymmetries. The results are encouraging and show that it will be feasible to evaluate the performance of the ATLAS detector with early EW data while preparing precision EW measurements. The "re-discovery" of the EW part of the Standard Model will begin with first data.

\section{References}

[1] G. Balossini et al., J. Phys. Conf. Ser. 110 (2008) 042002.

[2] W. K. Tung, H. L. Lai, A. Belyaev, J. Pumplin, D. Stump and C. P. Yuan, JHEP 0702 (2007) 053

[3] G. Aad et al. [ATLAS Collaboration], JINST 3 (2008) S08003

[4] G. Aad et al. [ATLAS Collaboration], CERN-OPEN-2008-020

[5] A. Tricoli, A. M. Cooper-Sarkar and C. Gwenlan, arXiv:hep-ex/0509002. 